Castello, A. P., J. A. Hurrell \& M. L. Pochettino. 2021. Estrategias metodológicas para acceder a la diversidad biocultural en huertos del periurbano platense (Buenos Aires, Argentina). Bonplandia 30(1): 5-26.

http://dx.doi.org/10.30972/bon.3014587

Recibido 21 Mayo 2020. Aceptado 8 Julio 2020.

Publicado en línea: 23 de diciembre 2020. Publicado impreso: 15 de febrero 2021.

ISSN 0524-0476 impreso. ISSN 1853-8460 en línea.

\title{
Estrategias Metodológicas PARA ACCEDER A la diVERSidAd biocultural EN HUERTOS del Periurbano platense (Buenos Aires, Argentina)
}

\author{
Methodological strategies to access the biocultural diversity in periurban homegardens of La \\ Plata (Buenos Aires, Argentina)
}

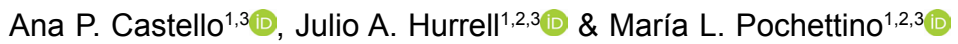

\begin{abstract}
Resumen: El estudio de los huertos es un tema relevante en etnobotánica, tanto por ser expresión de un conocimiento botánico local, como por la significativa diversidad biocultural que preservan. Esta es resultado de la experiencia vital de las y los horticultores y a menudo permanece oculta al emplear estrategias metodológicas cualitativas habituales en la disciplina. En esta contribución se utiliza por primera vez la aproximación biográfica en el cinturón hortícola platense (Buenos Aires, Argentina), espacio pluricultural y diverso en sus modos productivos, a fin de evaluar su potencial para conocer la diversidad biocultural de la zona. Al comparar los resultados con los obtenidos por técnicas sincrónicas de observación y entrevista, se identificó un número similar de taxones, con una ampliación del número total de plantas reconocidas y utilizadas en el área. Se observó que la experiencia previa adquirida en otras regiones habilitó el uso de ciertas plantas, no habitual en el área. Se discuten las ventajas y desventajas de la aproximación biográfica y el aporte de este tipo de estudios en proyectos de desarrollo local. Se concluye que la integración de metodologías sincrónicas con los estudios biográficos resulta en un abordaje integral y complejo de la diversidad biocultural que no podría alcanzarse por una única vía metodológica.
\end{abstract}

Palabras clave: Cinturón hortícola, entrevista, horticultura, observación, periurbano platense, trayectoria de vida.

Summary: The study of homegardens is an important subject in ethnobotany, as they are the expression of local botanical knowledge, and preserve significant biocultural diversity, This diversity is the result of the vital experience of horticulturalists, but it frequently remains hidden when usual ethnobotanical qualitative methodological strategies are applied. In this contribution, a biographical approach is used for the first time in the La Plata horticultural belt (Buenos Aires, Argentina), which is pluricultural and diverse in forms of production, to evaluate its usefulness for knowing the biocultural diversity of the area. When results are compared with those obtained by synchronous observation and interview techniques, a similar number of taxa was identified, with an increase of the total number of plants recognized and used in the area. It was observed that previous experience acquired in other regions enabled the use of certain plants, not habitual in the area. Both advantages and disadvantages of the biographical approach, as well as the contribution of this kind of studies in projects of local development are discussed. It is concluded that the integration of both synchronical methodologies with biographical studies derives in an integral and complex approach to biocultural diversity, not able to be achieved by a single methological way.

Key words: Horticultural belt, horticulture, interview, La Plata periurban area, life trajectory, observation.

${ }^{1}$ Laboratorio de Etnobotánica y Botánica Aplicada, Facultad de Ciencias Naturales y Museo, Universidad Nacional de La Plata.

${ }^{2}$ CONICET.

${ }^{3}$ E-mails: castelloap@gmail.com; juliohurrell@gmail.com; pochett@fcnym.unlp.edu.ar 


\section{Introducción}

Desde hace unos 30 años, los estudios etnobotánicos sobre los huertos se han incrementado en distintas partes del mundo (Lamont et al., 1999; Gaytán et al., 2001; Vogl et al., 2002; Vog1-Lukasser et al., 2002; Watson et Eyzaguirre, 2002; Blanckaert et al., 2004; Vogl-Lukasser et Vogl, 2004; Albuquerque et al., 2005; Das et Das, 2005; Huai et Hamilton, 2009; Vlkova et al., 2010; Calvet-Mir et al., 2011, 2014; Reyes-García et al., 2012; Pamungkas et al., 2013, Seta et al., 2013, Llobera Serra, 2014; Montañez-Escalante et al., 2014; Moyano Estrada, 2014; Gómez Sal et al., 2014, entre otros), y también en la Argentina, donde el Laboratorio de Etnobotánica y Botánica Aplicada (LEBA), de la Facultad de Ciencias Naturales y Museo, Universidad Nacional de La Plata, ha desarrollado una línea de investigación sobre el tema (Martínez et al., 2003; Lema, 2006; Pochettino et al., 2006, 2012, 2014, 2017; Turco et al., 2006; Del Río et al., 2007; Buet Costantino et al., 2010; Pochettino, 2010; Hurrell et al., 2011).

Según el Diccionario de la Real Academia Española (https://www.rae.es/), la "horticultura" es el cultivo (prácticas) en los "huertos", así como el arte que lo enseña. El creciente interés de la etnobotánica en el estudio de los huertos se debe a que las prácticas hortícolas permiten elucidar el conocimiento botánico que las orienta $\mathrm{y}$, a la vez, contribuyen a preservar la diversidad biocultural local (Pochettino et al., 2014). En especial, se ha enfatizado el estudio de los llamados "huertos familiares", por lo común, terrenos de poca extensión ubicados en las proximidades de las viviendas, cuya producción se destina al autoconsumo y, en ocasiones, a la comercialización a pequeña escala como suplemento para la economía doméstica (Hurrell et al., 2011; Pochettino et al., 2012, 2014). En los huertos familiares se encuentran especies y variedades, tanto cultivadas como espontáneas, utilizadas de diversas maneras. No obstante, esa diversidad no siempre es fácilmente registrada en nuestros estudios a partir de las metodologías más utilizadas: entrevistas y listados de plantas útiles reconocidas y utilizadas. Sin embargo, el motivo fundamental de esta problemática es la invisibilización de su importancia por parte de los propios productores, que ocurre cuando ciertas especies y variedades no registran un valor económico explícito. Estas entrarían en el concepto de "cosecha oculta" (Campbell et Luckert, 2002), acuñado por el Instituto Internacional para el Medioambiente y el Desarrollo (IIED, según su sigla en inglés) para destacar la importancia de las especies espontáneas en la subsistencia familiar.

En trabajos previos se destacó la importancia de la observación como estrategia para la visibilización y puesta en valor de la diversidad hortícola (Pochettino, 2010). En este caso, se aplicó un "enfoque biográfico", según técnicas registradas en Nazarea (1998), e inspiradas en la propia experiencia en el campo, donde las referencias al pasado personal de distintos sujetos eran recurrentes. A partir de estos comentarios surgieron los siguientes interrogantes: ¿Cómo las diversas experiencias de vida tienen un correlato en la diversidad de conocimientos botánicos de una persona?, ¿cómo estos saberes se actualizan en función de los contextos cambiantes que atraviesa el sujeto?

En ese marco, el objetivo de esta contribución es evaluar el potencial de los estudios biográficos para el registro de la biodiversidad local y su aplicación, con el objetivo a largo plazo de mantener e incrementar la diversidad biocultural a través de la revalorización de los conocimientos locales, que son múltiples y dinámicos.

\section{Materiales y Métodos}

Área de estudio. Huertos periurbanos platenses

En las proximidades de la ciudad de La Plata, capital de la provincia de Buenos Aires, Argentina, se encuentra una importante área productiva denominada "cinturón hortícola platense", localizada en el sector periurbano del área metropolitana local (Fig. 1), definido como una zona de transición entre los sectores netamente urbanos y 
rurales, con límites móviles según los ritmos de la urbanización (Barsky, 2010). Este "cinturón hortícola", además de ubicarse en el partido de La Plata, comprende espacios productivos ubicados en los partidos de Berisso, Ensenada y Berazategui, que abastecen de hortalizas, verduras y frutas frescas a la población del área metropolitana, y también de otras provincias argentinas (Benencia, 1997; Feito, 2007). En virtud de la tradición local, se consideran aquí "prácticas hortícolas" tanto las dedicadas a la producción de hortalizas y verduras como a la de frutas, por esto, las actividades frutihortícolas, se designan en este texto como "hortícolas" (Pochettino et al., 2014).

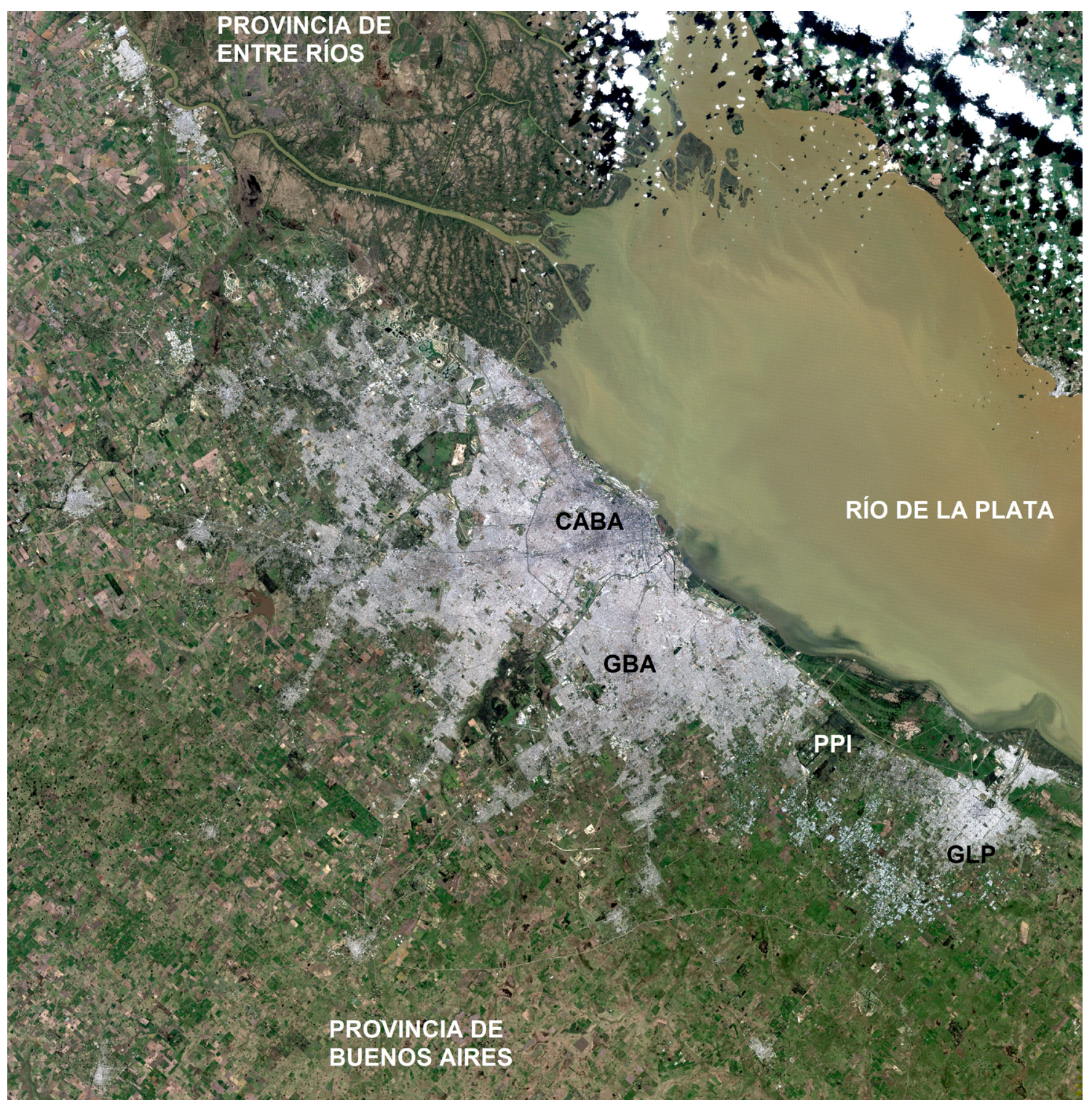

Fig. 1. Área de estudio (CABA: Ciudad Autónoma de Buenos Aires; GBA: Gran Buenos Aires; GLP: Gran La Plata; PPI: Parque Pereyra Iraola).

Fig. 1. Study area (CABA: Buenos Aires city; GBA: Greater Buenos Aires; GLP: Greater La Plata; PPI: Pereyra Iraola Park). 
La horticultura local se inició con la fundación de la ciudad de La Plata, a fines del siglo XIX, ligada al arribo de inmigrantes de diferentes países europeos que aportaron tanto sus conocimientos como sus prácticas tradicionales de origen (García, 2010; Hurrell et al., 2011). Buena parte de esta horticultura incipiente se vinculó al cultivo de la "uva americana", Vitis labrusca L., mediante el sistema de parral, adaptado a las condiciones locales, para la elaboración del denominado "vino de la costa" (por la proximidad de los huertos a la ribera del Río de la Plata), un producto considerado tradicional del área. Luego de un período de expansión inicial, los cultivos declinaron hasta el borde de la extinción; no obstante, presenta recientemente una interesante recuperación, gracias el esfuerzo de pobladores locales (Marasas et Velarde, 2000; Velarde et al., 2008; Hurrell et al., 2014).

La franja productiva platense presenta en la actualidad una elevada heterogeneidad, en cuanto al origen de los horticultores y a los tipos de huertos (familiares, comerciales), a la organización social del trabajo, a los estilos locales de producción, a los niveles de incorporación tecnológica y la conservación del material reproductivo. Esta heterogeneidad se refleja asimismo en las prácticas hortícolas, que a menudo implican la conservación de variedades tradicionales de la zona, como ocurre en los huertos de Isla Santiago (Ensenada), Isla Paulino y Los Talas (Berisso), localizados en la ribera del Río de la Plata (Hurrell et al., 2011), así como la incorporación de variedades hortícolas propias de tradiciones familiares de otras regiones (Pochettino et al., 2014). En este sentido, la zona es un espacio de diversidad cultural: descendientes de inmigrantes de fines del siglo XIX de origen suizo, italiano, español y eslavo; portugueses y japoneses llegados a mediados del siglo XX $\mathrm{y}$, desde hace unos veinte años, se produjo la incorporación de gran cantidad de inmigrantes bolivianos. Incluye también casos como los huertos enclavados en el Parque provincial y reserva de la biosfera Pereyra Iraola (PPI), "pulmón verde" entre el crecimiento urbano del Gran Buenos Aires y el del Gran La
Plata, con características particulares del acceso a la tierra (arriendo mediante canon al gobierno provincial), y donde se registran procesos de transformación productiva hacia la agroecología (Del Río et al., 2007).

\section{Aspectos metodológicos}

En los estudios realizados en el sector periurbano platense se aplicaron metodologías etnobotánicas cualitativas habituales, incluyendo la obtención del consentimiento previo informado para el desarrollo de la investigación y la difusión de sus resultados. Se procedió a la observación de los espacios hortícolas y de las prácticas cotidianas. Se realizaron listados libres y entrevistas abiertas y semiestructuradas (Albuquerque et al., 2014, 2019), referidas tanto a las plantas presentes en los huertos como a sus usos, con 25 interlocutores de ambos sexos y diferentes edades (de 28 a 82 años), a los que se accedió desde diferentes proyectos de extensión realizados previamente en la zona. Se trabajó a razón de un entrevistado por huerto, en los que se realizaron, además, colecciones de muestras, registros fotográficos, y obtención y procesamiento de ejemplares de herbario de referencia depositados para su documentación en herbarios reconocidos del área de estudio (BAA, BAB, LP, SI). Este material se identificó mediante caracteres de morfología externa e interna, en caso de ser necesario. Se presentan con sus nombres actualizados según The Plant List (2013).

Por otra parte, se aplicó el enfoque biográfico con una participante (M., 68 años), nacida en España, en la posguerra, quien emigró a Mendoza (Argentina) a los 12 años y, finalmente, se radicó en el Parque Pereyra Iraola en 2015. Este enfoque cualitativo, usualmente asociado a estudios históricos, sociológicos y antropológicos, intenta dar cuenta de un universo más amplio que el del individuo con el que se trabaja en un momento dado (entrevista). Se basa en entender a ese individuo socialmente constituido y en relación dialéctica con la estructura social local, con lo cual se posibilita pensar las experiencias individuales como producto y productoras de determinado grupo social (Bertaux, 1999; Ferraroti, 2007). 
En este caso, se ha recurrido a la "trayectoria de vida", en particular en relación a los conocimientos botánicos. La trayectoria de vida constituye una reconstrucción que hace el investigador a partir del relato de un sujeto sobre sus experiencias de vida en relación con el problema de investigación (Bertaux, 1999); en este caso, en relación a las variedades de plantas reconocidas y/o empleadas en distintos contextos a lo largo de su vida. Para el área de estudio, es la primera vez que esta metodología ha sido utilizada.

Cabe resaltar que esta estrategia presenta tanto potencialidades como dificultades que han sido planteadas por diversos autores (Bertaux, 1999; Ferraroti, 2007). Como aportes positivos podemos decir que prioriza el punto de vista del actor involucrado, permite abordar la dimensión temporal de los fenómenos, constituye una forma de resignificación de saberes para los interlocutores $\mathrm{y}$, además, puede ser una vía para generar hipótesis y nuevas preguntas de investigación.

Entre las dificultades, se puede destacar la que atañe a la selección de los interlocutores. Esto se debe a que es necesario que destinen más tiempo a los encuentros que con otras metodologías, deben tener una apertura para comunicar cuestiones que pueden ser consideradas personales, o íntimas, por lo cual hay que desarrollar una confianza previa entre el investigador y el interlocutor (Ferraroti, 2007) y, asimismo, dar cuenta de experiencias concretas en relación al tema y a las preguntas de investigación. También cabe preguntarse sobre cuán representativos son los interlocutores con respecto al grupo social o población de estudio: en este contexto, la representatividad es cualitativa (referida a los saberes), y no cuantitativa (numérica). Teniendo en cuenta las posibles dificultades planteadas, se considera conveniente aplicar el enfoque biográfico cuando el investigador ya tiene un conocimiento previo del área y de la temática (Nazarea, 1998). En este caso, la aplicación del enfoque biográfico, con la selección del interlocutor adecuado fue realizada teniendo con anterioridad un conocimiento del área y de los pobladores locales, a través de trabajos de investigación y de extensión universitaria.
La selección de la interlocutora en particular obedeció por una parte a su voluntad de participar en entrevistas de larga duración y su capacidad para recordar y poner en palabras sus memorias. Por otra parte, su historia de migraciones es semejante a la de la mayoría de horticultores del PPI que presentan una gran movilidad (Del Río et al., 2007). Por ello, la comparación de resultados obtenidos mediante este caso con técnicas sincrónicas de observación y entrevista permitió identificar continuidades y cambios en la diversidad biocultural local y resulta una propuesta para futuras investigaciones sobre el tema.

\section{Resultados}

La Tabla 1 presenta los distintos taxones relevados según las estrategias metodológicas empleadas. Mediante entrevistas y observación (sincrónicas) se registraron 98 taxones, por medio de la estrategia biográfica se registraron 99 taxones tanto presentes como "evocados", los cuales identificados taxonómicamente sobre la base de la descripción brindada por la interlocutora a partir de diversos estímulos "visuales" (fotografías, muestras, material de herbario), y "narrativos" (descripciones en distinto tipo de literatura). De este modo, se puede observar la incidencia de la experiencia personal en el incremento del total de los saberes botánicos locales teniendo en cuenta que hay 38 taxones que fueron registrados mediante los dos tipos de abordaje (Fig. 2). Los 159 taxones registrados incluyen especies, subespecies o variedades botánicas, reconocidos como diferentes por los pobladores locales (Fig. 3). La gran mayoría de las plantas relevadas se utilizan con fines alimentarios; no obstante, se incluyeron también otras con distintos usos (medicinal, artesanal) (Fig. 4), dado que forman parte del repertorio de taxones presentes en los huertos.

En la Tabla 2 se resume la información registrada exclusivamente mediante la trayectoria de vida, donde se observa la incorporación de nuevos saberes hortícolas y su actualización en distintos contextos. 
Tabla 1. Taxones registrados en las huertas del periurbano platense según distintas estrategias metodológicas (E-O: entrevista y observación) (El símbolo • indica presencia).

Table 1. Taxa recorded in homegardens of La Plata outskirts by means of different methodological strategies (E-O: interview and observation) (The symbol $\bullet$ means presence).

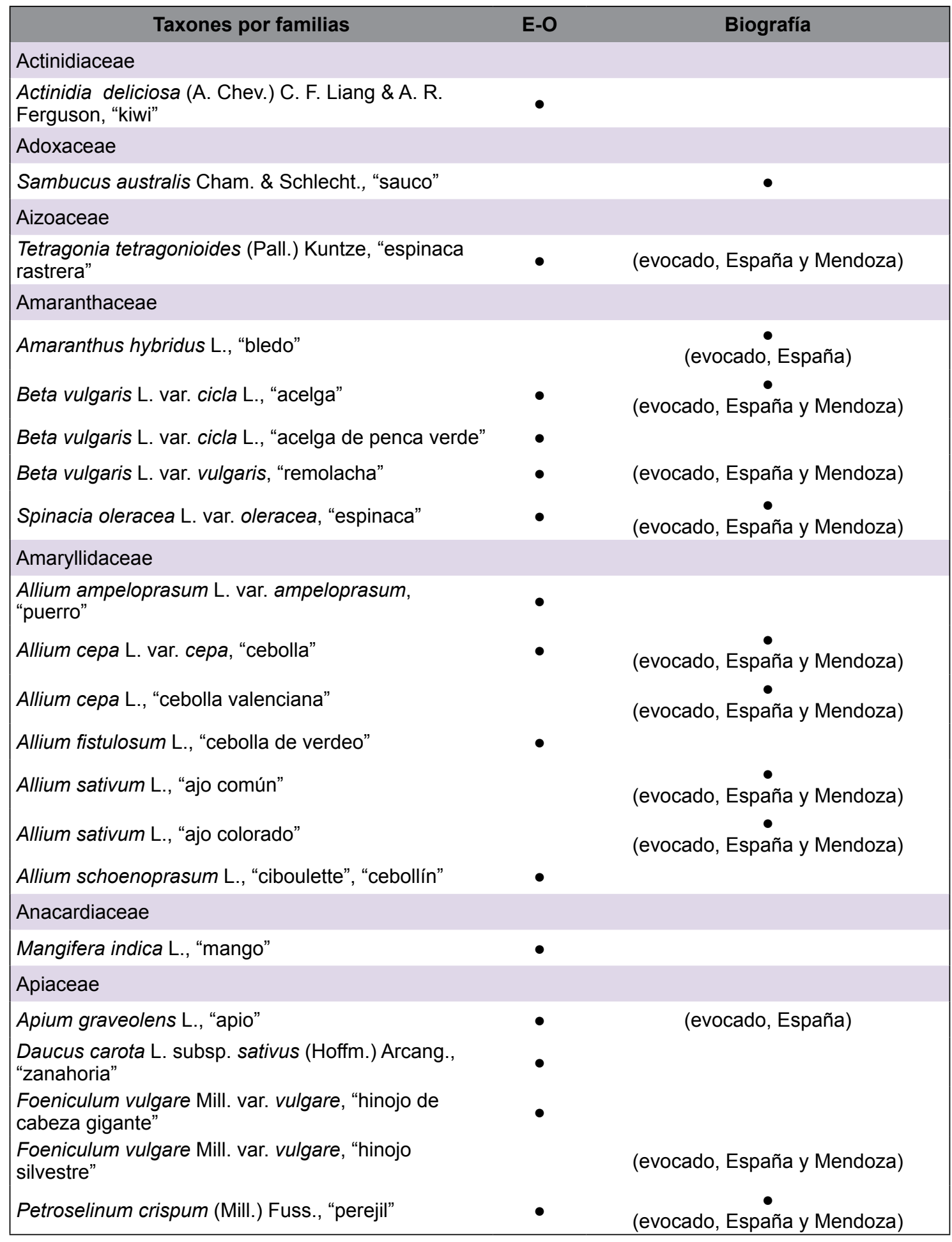




\begin{tabular}{|c|c|c|}
\hline Taxones por familias & E-O & Biografía \\
\hline \multicolumn{3}{|l|}{ Araliaceae } \\
\hline Tetrapanax papyrifer (Hook.) K. Koch, "ambay" & $\bullet$ & \\
\hline \multicolumn{3}{|l|}{ Asparagaceae } \\
\hline Asparagus officinalis L., "espárrago" & & (evocado, Mendoza) \\
\hline \multicolumn{3}{|l|}{ Asteraceae } \\
\hline Artemisia absinthium L., "ajenjo" & $\bullet$ & \\
\hline Artemisia verlotorum Lamotte, "sertal" & & $\bullet$ \\
\hline Baccharis articulata (Lam.) Pers., "carqueja" & & $\bullet$ \\
\hline Baccharis salicifolia (Ruiz \& Pav.) Pers., "chilca" & & (evocado, Mendoza) \\
\hline Calendula officinalis L., "caléndula" & $\bullet$ & \\
\hline Carduus sp., "cardo común" & & (evocado, España) \\
\hline Cichorium endivia L., "escarola" & $\bullet$ & \\
\hline Cichorium intybus L., "achicoria" & $\bullet$ & \\
\hline Cichorium intybus L., "escarola silvestre" & & $\bullet$ \\
\hline $\begin{array}{l}\text { Cynara cardunculus L. var. scolymus (L.) Fiori, } \\
\text { "alcaucil" }\end{array}$ & $\bullet$ & (evocado, España y Mendoza) \\
\hline Cynara cardunculus L., "cardo de Castilla" & & (evocado, España) \\
\hline Lactuca sativa L., "lechuga” & $\bullet$ & \\
\hline Matricaria chamomilla L., "manzanilla" & - & (evocado, España) \\
\hline Sonchus oleraceus L., "cerraja" & & (evocado, Mendoza) \\
\hline Taraxacum officinale L., "diente de león" & $\bullet$ & \\
\hline \multicolumn{3}{|l|}{ Boraginaceae } \\
\hline Borago officinalis L., "borraja" & $\bullet$ & \\
\hline \multicolumn{3}{|l|}{ Brassicaceae } \\
\hline Brassica napus L., "grilo" & $\bullet$ & \\
\hline Brassica napus L., "nabiza" & & (evocado, España) \\
\hline Brassica oleracea L. var. capitata L., "repollo" & $\bullet$ & \\
\hline Brassica oleracea L. var. italica L., "brócoli” & $\bullet$ & \\
\hline Brassica rapa L. var. rapa, "nabo" & & (evocado, España y Mendoza) \\
\hline Eruca vesicaria (L.) Cav., "rúcula" & $\bullet$ & \\
\hline Raphanus sativus L. var. sativus, "rabanito" & $\bullet$ & \\
\hline \multicolumn{3}{|l|}{ Caryophyllaceae } \\
\hline Silene vulgaris (Moench.) Garcke, "colleja" & & (evocado, España) \\
\hline \multicolumn{3}{|l|}{ Convolvulaceae } \\
\hline Convolvulus arvensis L., "correhuela" & & $\bullet$ \\
\hline
\end{tabular}




\begin{tabular}{|c|c|c|}
\hline Taxones por familias & E-O & Biografía \\
\hline Ipomoea batatas (L.) Lam., "batata" & $\bullet$ & (evocado, España y Mendoza) \\
\hline Ipomoea batatas (L.) Lam., "batata blanca" & $\bullet$ & \\
\hline \multicolumn{3}{|l|}{ Cucurbitaceae } \\
\hline $\begin{array}{l}\text { Citrullus lanatus (Thunb.) Matsum. \& Nakai "sandía } \\
\text { roja" }\end{array}$ & $\bullet$ & \\
\hline Cucumis melo L. subsp. melo, "melón" & $\bullet$ & \\
\hline Cucumis sativus L., "pepino" & $\bullet$ & (evocado, España y Mendoza) \\
\hline Cucurbita ficifolia Bouché, "cayote" & $\bullet$ & \\
\hline $\begin{array}{l}\text { Cucurbita maxima Duchesne subsp. maxima, } \\
\text { "zapallo hongo" }\end{array}$ & $\bullet$ & \\
\hline $\begin{array}{l}\text { Cucurbita maxima Duchesne subsp. maxima, } \\
\text { "zapallo plomo" }\end{array}$ & & (evocado, Mendoza) \\
\hline $\begin{array}{l}\text { Cucurbita maxima Duchesne var. zapallito (Carrière) } \\
\text { Millán, "zapallito" }\end{array}$ & $\bullet$ & \\
\hline $\begin{array}{l}\text { Cucurbita maxima Duchesne var. zapallito (Carriére) } \\
\text { Millán, "zapallito lustroso" }\end{array}$ & $\bullet$ & \\
\hline Cucurbita moschata Duchesne, "zapallo batata" & & (evocado, Mendoza) \\
\hline Cucurbita pepo L., "anco" & $\bullet$ & \\
\hline Cucurbita pepo L., "zapallo de brujita" & & (evocado, España y Mendoza) \\
\hline Cucurbita pepo L., "zapallo verde oscuro" & & (evocado, España y Mendoza) \\
\hline Cucurbita pepo L., "zapallo cantimplora" & & (evocado, España y Mendoza) \\
\hline Lagenaria siceraria (Molina) Standl., "mate" & $\bullet$ & \\
\hline Sechium edule (Jacq.) Sw., "papa del aire" & $\bullet$ & \\
\hline \multicolumn{3}{|l|}{ Ebenaceae } \\
\hline Diospyros kaki L. f., "caqui" & $\bullet$ & (evocado, España y Mendoza) \\
\hline \multicolumn{3}{|l|}{ Fabaceae } \\
\hline Galega officinalis L., "alfalfa gallega" & & (evocado, España) \\
\hline Lablab purpureus (L.) Sweet, "poroto japonés" & $\bullet$ & (evocado, España) \\
\hline Lens culinaris Medik., "lenteja" & & (evocado, España) \\
\hline Phaseolus lunatus L., "poroto de manteca" & $\bullet$ & \\
\hline Phaseolus vulgaris L. var. vulgaris, "poroto" & $\bullet$ & \\
\hline Phaseolus vulgaris L. var. vulgaris, "poroto alubia" & & (evocado, España) \\
\hline Phaseolus vulgaris L. var. vulgaris, "poroto blanco" & & (evocado, España) \\
\hline Phaseolus vulgaris L. var. vulgaris, "poroto negro" & & (evocado, España) \\
\hline Phaseolus vulgaris L. var. vulgaris, "chaucha" & & (evocado, España) \\
\hline $\begin{array}{l}\text { Phaseolus vulgaris L. var. vulgaris, "chaucha } \\
\text { manteca" }\end{array}$ & & (evocado, Mendoza) \\
\hline Pisum sativum L., "arveja" & $\bullet$ & (evocado, España y Mendoza) \\
\hline
\end{tabular}




\begin{tabular}{|c|c|c|}
\hline Taxones por familias & E-O & Biografía \\
\hline Vicia faba L., "haba" & $\bullet$ & (evocado. España y Mendoza) \\
\hline $\begin{array}{l}\text { Vigna unquiculata (L.) Walp. ssp. sesquipedalis (L.) } \\
\text { Verdc., "chaucha metro" }\end{array}$ & & (evocado, Mendoza) \\
\hline \multicolumn{3}{|l|}{ Fagaceae } \\
\hline Castanea sativa Mill., "castaño" & & (evocado, España) \\
\hline Quercus spp., "roble" & & (evocado, Mendoza) \\
\hline \multicolumn{3}{|l|}{ Juglandaceae } \\
\hline Carya illinoinensis (Wangenh.) K. Koch, "pecán" & • & \\
\hline Juglans regia L., "nuez de Castilla" & & (evocado, España) \\
\hline \multicolumn{3}{|l|}{ Lamiaceae } \\
\hline Lavandula angustifolia Mill., "lavanda" & $\bullet$ & \\
\hline Melissa officinalis L., "melisa" & $\bullet$ & \\
\hline Mentha spicata L., "menta" & $\bullet$ & $\bullet$ \\
\hline Mentha x piperita L., "menta peperina" & & $\bullet$ \\
\hline Ocimum basilicum L., "albahaca" & $\bullet$ & (evocado, España y Mendoza) \\
\hline Origanum vulgare L., "orégano" & $\bullet$ & (evocado, España) \\
\hline Origanum majorana L., "mejorana" & & (evocado, España) \\
\hline Rosmarinus officinalis L., "romero" & $\bullet$ & (evocado, España y Mendoza) \\
\hline Salvia officinalis L., "salvia" & $\bullet$ & \\
\hline Thymus vulgaris L., "tomillo" & $\bullet$ & (evocado, España y Mendoza) \\
\hline \multicolumn{3}{|l|}{ Lauraceae } \\
\hline Laurus nobilis L., "laurel" & $\bullet$ & (evocado, Mendoza) \\
\hline Persea americana Mill., "palta" & $\bullet$ & \\
\hline \multicolumn{3}{|l|}{ Lythraceae } \\
\hline Punica granatum L., "granada" & $\bullet$ & \\
\hline \multicolumn{3}{|l|}{ Malvaceae } \\
\hline Malva parviflora L., "malva" & & (evocado, España) \\
\hline \multicolumn{3}{|l|}{ Moraceae } \\
\hline Ficus carica L., "higo" & $\bullet$ & \\
\hline Morus alba L., "mora blanca" & & $\bullet$ \\
\hline Morus alba L., "mora rosada" & & $\bullet$ \\
\hline Morus nigra L., "mora negra" & & $\bullet$ \\
\hline \multicolumn{3}{|l|}{ Musaceae } \\
\hline Musa x paradisiaca L., "banana" & $\bullet$ & \\
\hline \multicolumn{3}{|l|}{ Myrtaceae } \\
\hline Eucalyptus cinerea F. Muell. ex Benth., "eucalipto" & $\bullet$ & \\
\hline
\end{tabular}




\begin{tabular}{|c|c|c|}
\hline Taxones por familias & E-O & Biografía \\
\hline Eucalyptus globulus Labill., "eucalipto" & $\bullet$ & (evocado, Mendoza) \\
\hline \multicolumn{3}{|l|}{ Oleaceae } \\
\hline Olea europea L., "olivo" & $\bullet$ & (evocado, España y Mendoza) \\
\hline \multicolumn{3}{|l|}{ Papaveraceae } \\
\hline Papaver sp., "amapola" & & (evocado, España) \\
\hline \multicolumn{3}{|l|}{ Poaceae } \\
\hline Cymbopogon citratus (DC.) Stapf, "pasto limón" & $\bullet$ & \\
\hline Triticum aestivum, "trigo" & & (evocado, España) \\
\hline Zea mays L., "maíz" & $\bullet$ & (evocado, España y Mendoza) \\
\hline \multicolumn{3}{|l|}{ Polygonaceae } \\
\hline $\begin{array}{l}\text { Rumex crispus L., "lengua de buey", "lengua de } \\
\text { vaca" }\end{array}$ & $\bullet$ & (evocado, Mendoza) \\
\hline \multicolumn{3}{|l|}{ Portulacaceae } \\
\hline Portulaca oleracea L., "verdolaga" & $\bullet$ & (evocado, Mendoza) \\
\hline \multicolumn{3}{|l|}{ Rosaceae } \\
\hline Cydonia oblonga Mill., "membrillo" & $\bullet$ & \\
\hline Eriobotrya japonica (Thunb.) Lindl., "níspero" & - & (evocado, España) \\
\hline Fragaria x ananassa (Weston) Duchesne, "frutilla" & $\bullet$ & \\
\hline Malus pumila Mill., "manzana" & $\bullet$ & \\
\hline Malus pumila Mill., "manzana roja" & & (evocado, España y Mendoza) \\
\hline Malus pumila Mill., "manzana verde" & & (evocado, Mendoza) \\
\hline Prunus amygdalus Batsch, "almendra" & $\bullet$ & (evocado, España) \\
\hline Prunus avium (L.) L., "cereza" & - & (evocado, España) \\
\hline Prunus cerasus L., "guinda" & • & (evocado, España) \\
\hline Prunus domestica L., "ciruela" & • & (evocado, España y Mendoza) \\
\hline Prunus persica Batsch., "durazno" & $\bullet$ & (evocado, España y Mendoza) \\
\hline Pyrus communis L., "pera" & $\bullet$ & (evocado, España y Mendoza) \\
\hline Rubus ulmifolius Schott., "zarzamora" & & (evocado, España) \\
\hline \multicolumn{3}{|l|}{ Rutaceae } \\
\hline Citrus japonicaThunb., "quinoto" & $\bullet$ & \\
\hline Citrus x aurantium L. Grupo Naranjo Dulce, "naranja" & $\bullet$ & \\
\hline Citrus x aurantium L. Grupo Pomelo, "pomelo" & $\bullet$ & \\
\hline Citrus x limon (L.) Osbeck, "limón" & $\bullet$ & \\
\hline Citrus reticulata Blanco, "mandarina" & $\bullet$ & \\
\hline \multicolumn{3}{|l|}{ Solanaceae } \\
\hline Capsicum annuum L., "ají chiquito" & & (evocado, Mendoza) \\
\hline
\end{tabular}




\begin{tabular}{|c|c|c|}
\hline Taxones por familias & E-O & Biografía \\
\hline Capsicum annuum L., "ají picante" & & (evocado, España) \\
\hline Capsicum annuum L., "ají redondo" & & (evocado, Mendoza) \\
\hline Capsicum annuum L., "ají siete colores" & & (evocado, Mendoza) \\
\hline Capsicum annuum L., "morrón" & $\bullet$ & \\
\hline Capsicum annuum L., "pimiento o morrón dulce" & & (evocado, España) \\
\hline Capsicum annuum L., "pimiento amarillo o banana" & & (evocado, Mendoza) \\
\hline Capsicum annuum L., "pimiento calahorra" & & (evocado, Mendoza) \\
\hline Capsicum annuum L., "pimiento cacho de cabra" & & (evocado, Mendoza) \\
\hline Capsicum annuum L., "pimiento carnoso" & & (evocado, Mendoza) \\
\hline Capsicum annuum L., "pimiento medio kilo" & & (evocado, Mendoza) \\
\hline $\begin{array}{l}\text { Capsicum baccatum L. var. pendulum (Willd.) } \\
\text { Eshbaugh, "campanita" }\end{array}$ & - & (evocado, Mendoza) \\
\hline Nicotiana tabacum L., "tabaco" & $\bullet$ & \\
\hline Solanum lycopersicum L. var. lycopersicum, "tomate" & $\bullet$ & (evocado, España y Mendoza) \\
\hline Solanum lycopersicum L., "tomate amarillo" & & (evocado, Mendoza) \\
\hline Solanum lycopersicum L., "tomate de racimo" & & (evocado, Mendoza) \\
\hline Solanum lycopersicum L., "tomate perita" & & (evocado, Mendoza) \\
\hline Solanum lycopersicum L., "tomate Río Grande" & & (evocado, Mendoza) \\
\hline Solanum melongena L., "berenjena" & $\bullet$ & \\
\hline Solanum tuberosum L. subsp. tuberosum, "papa” & • & (evocado, España y Mendoza) \\
\hline \multicolumn{3}{|l|}{ Urticaceae } \\
\hline Parietaria judaica L., "buscapina" & & $\bullet$ \\
\hline Urtica urens L., "ortiga" & $\bullet$ & (evocado, España) \\
\hline \multicolumn{3}{|l|}{ Verbenaceae } \\
\hline Aloysia citriodora Palau, "cedrón" & $\bullet$ & \\
\hline Aloysia polystachya (Griseb.) Moldenke, "burrito" & $\bullet$ & \\
\hline Lippia turbinata Griseb., "poleo" & $\bullet$ & \\
\hline \multicolumn{3}{|l|}{ Vitaceae } \\
\hline Vitis labrusca L., "uva chinche", "uva Isabella" & $\bullet$ & \\
\hline Vitis vinifera L., "uva europea" & $\bullet$ & (evocado, España y Mendoza) \\
\hline \multicolumn{3}{|l|}{ Xanthorrhoeaceae } \\
\hline Aloe arborescens Mill., "aloe" & $\bullet$ & \\
\hline Aloe vera (L.) Burm. f., "aloe" & • & \\
\hline
\end{tabular}


BONPLANDIA 30(1). 2021

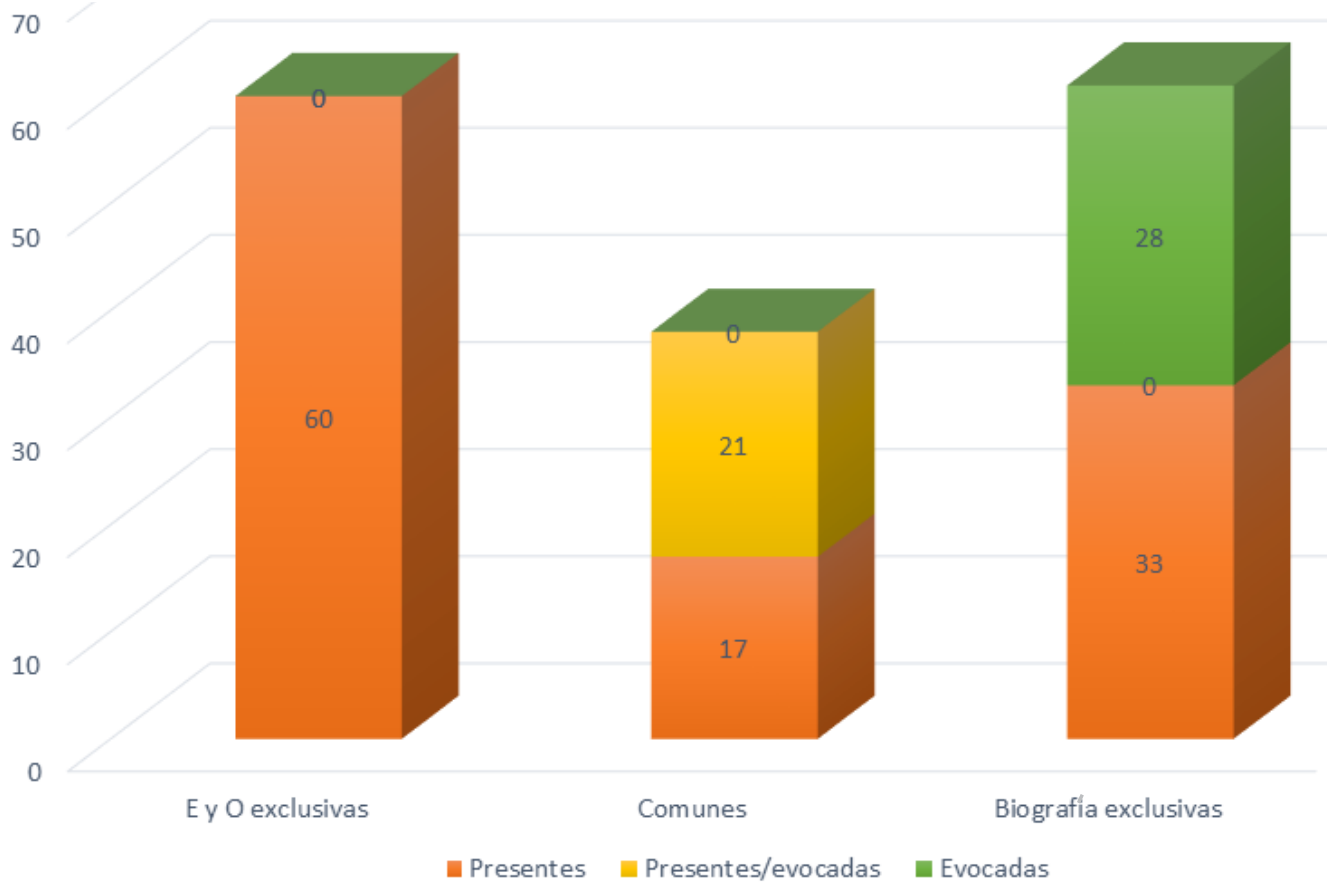

Fig. 2. Comparación del número de taxones reconocidos por los pobladores locales y registrados según las distintas estrategias metodológicas utilizadas.

Fig. 2. Comparison of the number of taxa recognized by local people and recorded by means of the diverse methodological strategies employed.

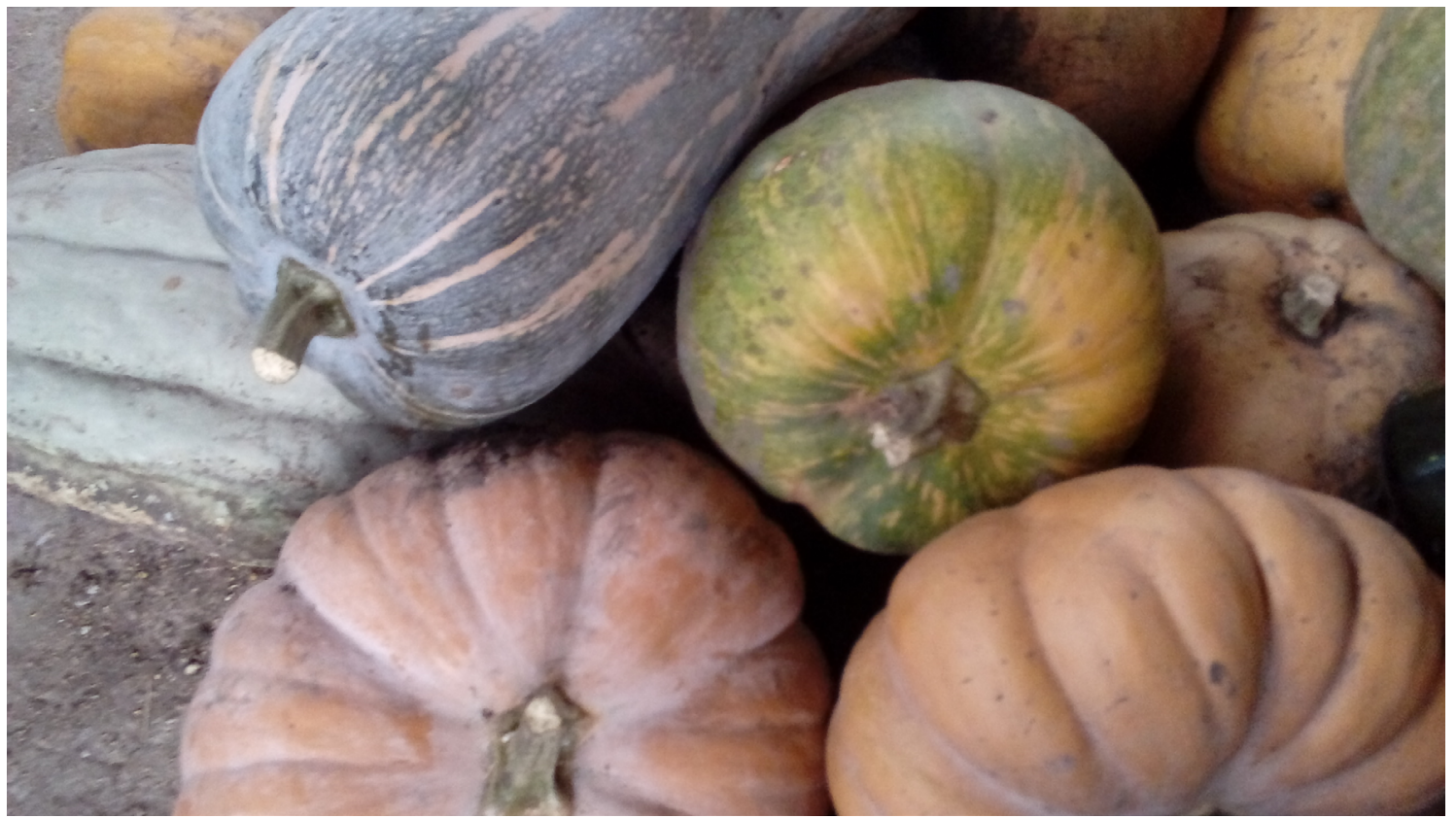

Fig. 3. Distintas variedades de Cucurbita moschata cultivadas en el PPI.

Fig. 3. Different varieties of Cucurbita moschata grown in PPI. 


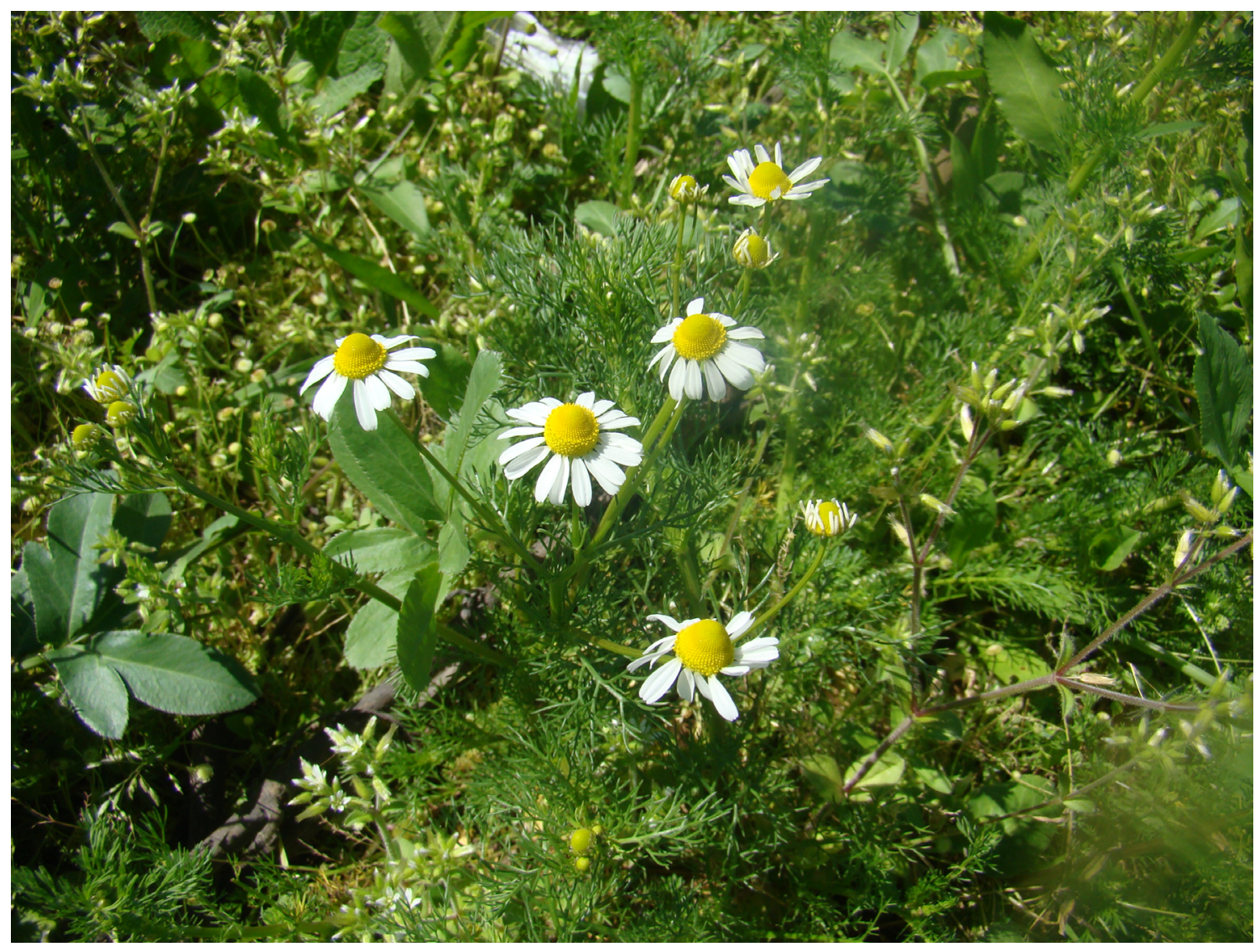

Fig. 4. "Manzanilla" (Matricaria chamomilla) de uso terapéutico en PPI.

Fig. 4. "Manzanilla" (Matricaria chamomilla) used as therapeutic in PPI.

Tabla 2. Conocimiento botánico de una interlocutora, registrado mediante la estrategia de trayectoria de vida. Table 2. Botanical knowledge of one of the interviewees, recorded by means of life trajectory strategy.

\begin{tabular}{|c|c|c|c|c|}
\hline $\begin{array}{l}\text { Nombre } \\
\text { atribuido }\end{array}$ & $\begin{array}{l}\text { Modo de } \\
\text { obtención }\end{array}$ & Formas de utilización & Destino & Lugar de uso \\
\hline Acelga & Cultivo & Alimenticio & Autoconsumo & $\begin{array}{l}\text { España - } \\
\text { Mendoza - PPI }\end{array}$ \\
\hline Ají 7 colores & Cultivo & $\begin{array}{l}\text { Alimenticio: fresco, en conserva o } \\
\text { seco para hacer pimentón picante }\end{array}$ & Autoconsumo & Mendoza \\
\hline Ají Campanita & Cultivo & $\begin{array}{l}\text { Alimento: fresco, en conserva o seco } \\
\text { para hacer pimentón picante }\end{array}$ & Autoconsumo & Mendoza - PPI \\
\hline $\begin{array}{l}\text { Ají Chiquito, } \\
\text { alargado y } \\
\text { finito }\end{array}$ & Cultivo & $\begin{array}{l}\text { Alimenticio: fresco, en conserva o } \\
\text { seco para hacer pimentón picante }\end{array}$ & Autoconsumo & Mendoza \\
\hline Ají picante & Cultivo & Alimenticio & Autoconsumo & España \\
\hline Ají Redondo & Cultivo & $\begin{array}{l}\text { Alimenticio: fresco, en conserva o } \\
\text { seco para hacer pimentón picante }\end{array}$ & Autoconsumo & Mendoza \\
\hline Ajo Colorado & Cultivo & Alimenticio & Autoconsumo & $\begin{array}{l}\text { España - } \\
\text { Mendoza - PPI }\end{array}$ \\
\hline
\end{tabular}




\begin{tabular}{|c|c|c|c|c|}
\hline $\begin{array}{l}\text { Nombre } \\
\text { atribuido }\end{array}$ & $\begin{array}{l}\text { Modo de } \\
\text { obtención }\end{array}$ & Formas de utilización & Destino & Lugar de uso \\
\hline Ajo común & Cultivo & Alimenticio & Autoconsumo & $\begin{array}{l}\text { España - } \\
\text { Mendoza - PPI }\end{array}$ \\
\hline Albahaca & Cultivo & $\begin{array}{l}\text { Alimenticio } \\
\text { /condimenticia. Ahuyenta insectos en } \\
\text { huerta }\end{array}$ & Autoconsumo & $\begin{array}{l}\text { España - } \\
\text { Mendoza - PPI }\end{array}$ \\
\hline Alcaucil & Recolección & Alimenticio: cocido o en conserva & Autoconsumo & $\begin{array}{l}\text { España - } \\
\text { Mendoza }\end{array}$ \\
\hline Alfalfa gallega & Recolección & Alimenticio: raíz hervida, hojas, flores & Autoconsumo & España - PPI \\
\hline Almendra & Recolección & Alimenticio & Autoconsumo & España \\
\hline Amapola & Recolección & $\begin{array}{l}\text { Medicinal: para facilitar el sueño, } \\
\text { tranquilizante }\end{array}$ & Autoconsumo & España \\
\hline Apio & Recolección & Alimenticio & Autoconsumo & Mendoza \\
\hline Arveja & Cultivo & Alimenticio & $\begin{array}{l}\text { Autoconsumo } \\
\text { Trabajo } \\
\text { asalariado: } \\
\text { fábrica de } \\
\text { conservas } \\
\text { (Mendoza) }\end{array}$ & $\begin{array}{l}\text { España - } \\
\text { Mendoza }\end{array}$ \\
\hline Batata & Cultivo & Alimenticio & Autoconsumo & $\begin{array}{l}\text { España - } \\
\text { Mendoza - PPI }\end{array}$ \\
\hline Bledo & Recolección & Alimenticio & Autoconsumo & Mendoza - PPI \\
\hline Buscapina & Cultivo & Medicinal: para problemas digestivos & Autoconsumo & PPI \\
\hline Caqui & $\begin{array}{l}\text { Recolección } \\
\text { Cultivo }\end{array}$ & Alimenticio & Autoconsumo & $\begin{array}{l}\text { España - } \\
\text { Mendoza }\end{array}$ \\
\hline Cardo común & Recolección & $\begin{array}{l}\text { Alimenticio: tallo hervido y flores } \\
\text { tiernas }\end{array}$ & Autoconsumo & España - PPI \\
\hline $\begin{array}{l}\text { Cardo de } \\
\text { Castilla }\end{array}$ & Recolección & $\begin{array}{l}\text { Alimenticio: tallo hervido y flores } \\
\text { tiernas }\end{array}$ & Autoconsumo & España - PPI \\
\hline Carqueja & Cultivo & Medicinal: para problemas del hígado & Autoconsumo & PPI \\
\hline Castaño & Recolección & Utensilio: tallado de cucharas & Autoconsumo & España \\
\hline $\begin{array}{l}\text { Cebolla } \\
\text { común }\end{array}$ & Cultivo & Alimenticio & Autoconsumo & $\begin{array}{l}\text { España - } \\
\text { Mendoza - PPI }\end{array}$ \\
\hline $\begin{array}{l}\text { Cebolla } \\
\text { valenciana }\end{array}$ & Cultivo & Alimenticio & Autoconsumo & $\begin{array}{l}\text { España - } \\
\text { Mendoza - PPI }\end{array}$ \\
\hline Cereza & Recolección & $\begin{array}{l}\text { Alimenticio: fruta fresca o en } \\
\text { conservas dulces }\end{array}$ & Autoconsumo & España \\
\hline Cerraja & Recolección & Alimenticio: hojas & Autoconsumo & Mendoza - PPI \\
\hline Chaucha & Cultivo & Alimenticio: en guiso, secas & Autoconsumo & España \\
\hline $\begin{array}{l}\text { Chaucha } \\
\text { Japonesa }\end{array}$ & Cultivo & Alimenticio: en guiso, secas & Autoconsumo & $\begin{array}{l}\text { Mendoza - } \\
\text { Parque Pereyra } \\
\text { Iraola }\end{array}$ \\
\hline $\begin{array}{l}\text { Chaucha } \\
\text { manteca }\end{array}$ & Cultivo & Alimenticio: en guiso, secas & Autoconsumo & Mendoza \\
\hline $\begin{array}{l}\text { Chaucha } \\
\text { metro }\end{array}$ & Cultivo & Alimenticio: en guiso, secas & Autoconsumo & Mendoza \\
\hline Chilca & Recolección & $\begin{array}{l}\text { Medicinal: para problemas } \\
\text { respiratorios, expectorante }\end{array}$ & Autoconsumo & Mendoza - PPI \\
\hline
\end{tabular}




\begin{tabular}{|c|c|c|c|c|}
\hline $\begin{array}{l}\text { Nombre } \\
\text { atribuido }\end{array}$ & $\begin{array}{l}\text { Modo de } \\
\text { obtención }\end{array}$ & Formas de utilización & Destino & Lugar de uso \\
\hline Ciruela & Recolección & $\begin{array}{l}\text { Alimenticio: fruta fresca, seca o en } \\
\text { conservas dulces }\end{array}$ & $\begin{array}{l}\text { Autoconsumo } \\
\text { Trabajo } \\
\text { asalariado: } \\
\text { en cosecha } \\
\text { y en fábrica } \\
\text { de conservas } \\
\text { (Mendoza) }\end{array}$ & $\begin{array}{l}\text { España - } \\
\text { Mendoza }\end{array}$ \\
\hline Colleja & Recolección & $\begin{array}{l}\text { Alimenticio: en ensalada, guiso y } \\
\text { tortilla }\end{array}$ & Autoconsumo & España \\
\hline Correhuela & Recolección & $\begin{array}{l}\text { Alimenticio: hojas. Consumo humano } \\
\text { y animal }\end{array}$ & Autoconsumo & PPI \\
\hline Durazno & Recolección & $\begin{array}{l}\text { Alimenticio: fruta fresca, seca o en } \\
\text { conservas dulces }\end{array}$ & $\begin{array}{l}\text { Autoconsumo } \\
\text { Trabajo } \\
\text { asalariado: } \\
\text { en cosecha } \\
\text { y en fábrica } \\
\text { de conservas } \\
\text { (Mendoza) }\end{array}$ & $\begin{array}{l}\text { España - } \\
\text { Mendoza }\end{array}$ \\
\hline $\begin{array}{l}\text { Escarola } \\
\text { silvestre }\end{array}$ & Recolección & Alimenticio: hojas & Autoconsumo & PPI \\
\hline Espárrago & Recolección & Alimenticio & Autoconsumo & Mendoza \\
\hline $\begin{array}{l}\text { Espinaca } \\
\text { común }\end{array}$ & Cultivo & Alimenticio: fresco o cocido & Autoconsumo & $\begin{array}{l}\text { España - } \\
\text { Mendoza - PPI }\end{array}$ \\
\hline $\begin{array}{l}\text { Espinaca } \\
\text { rastrera }\end{array}$ & Cultivo & Alimenticio: fresco o cocido & Autoconsumo & $\begin{array}{l}\text { España - } \\
\text { Mendoza }\end{array}$ \\
\hline Eucalipto & Recolección & $\begin{array}{l}\text { Medicinal: para problemas } \\
\text { respiratorios, expectorante en jarabe }\end{array}$ & Autoconsumo & Mendoza - PPI \\
\hline Guinda & Recolección & $\begin{array}{l}\text { Alimenticio: fruta fresca o en } \\
\text { conservas dulces }\end{array}$ & Autoconsumo & España \\
\hline Haba & Cultivo & $\begin{array}{l}\text { Alimenticio: cocidas en ensalada, } \\
\text { tortilla, guiso, etc. o secas }\end{array}$ & Autoconsumo & $\begin{array}{l}\text { España - } \\
\text { Mendoza - PPI }\end{array}$ \\
\hline $\begin{array}{l}\text { Hinojo } \\
\text { silvestre }\end{array}$ & Recolección & Alimenticio & Autoconsumo & $\begin{array}{l}\text { España - } \\
\text { Mendoza }\end{array}$ \\
\hline Laurel & Recolección & $\begin{array}{l}\text { Medicinal: para problemas } \\
\text { respiratorios, expectorante en jarabe }\end{array}$ & Autoconsumo & Mendoza-PPI \\
\hline $\begin{array}{l}\text { Lengua } \\
\text { de Buey o } \\
\text { Lengua de } \\
\text { Vaca }\end{array}$ & Recolección & Alimenticio: hojas & Autoconsumo & Mendoza - PPI \\
\hline Lenteja & $\begin{array}{l}\text { Entrega por } \\
\text { parte del } \\
\text { gobierno }\end{array}$ & Alimenticio: en guisos & Autoconsumo & España \\
\hline Maíz & Cultivo & $\begin{array}{l}\text { Alimenticio } \\
\text { La chalas como envoltura de } \\
\text { cigarrillos (sólo España) }\end{array}$ & Autoconsumo & $\begin{array}{l}\text { España - } \\
\text { Mendoza - PPI }\end{array}$ \\
\hline Malva & $\begin{array}{l}\text { Recolección } \\
\text { (España) } \\
\text { Cultivo } \\
\text { (Pereyra) }\end{array}$ & $\begin{array}{l}\text { Medicinal: baños locales para } \\
\text { estreñimientos y hemorroides }\end{array}$ & Autoconsumo & España - PPI \\
\hline Manzana roja & Recolección & Alimenticio & Autoconsumo & $\begin{array}{l}\text { España - } \\
\text { Mendoza }\end{array}$ \\
\hline
\end{tabular}




\begin{tabular}{|c|c|c|c|c|}
\hline $\begin{array}{l}\text { Nombre } \\
\text { atribuido }\end{array}$ & $\begin{array}{l}\text { Modo de } \\
\text { obtención }\end{array}$ & Formas de utilización & Destino & Lugar de uso \\
\hline $\begin{array}{l}\text { Manzana } \\
\text { verde }\end{array}$ & & Alimenticio & Autoconsumo & Mendoza \\
\hline Manzanilla & Recolección & $\begin{array}{l}\text { Medicinal: té digestivo y tranquilizante } \\
\text { Fumado, como sustituto de tabaco }\end{array}$ & Autoconsumo & España - PPI \\
\hline Mejorana & Recolección & Medicinal & Comercialización & España \\
\hline Menta & Cultivo & Medicinal: para problemas digestivos & Autoconsumo & PPI \\
\hline $\begin{array}{l}\text { Menta } \\
\text { Peperina }\end{array}$ & Cultivo & Medicinal: para problemas digestivos & Autoconsumo & PPI \\
\hline Mora Blanca & Recolección & $\begin{array}{l}\text { Alimenticio: fruto fresco o en } \\
\text { mermelada }\end{array}$ & Autoconsumo & PPI \\
\hline Mora Negra & Recolección & $\begin{array}{l}\text { Alimenticio: fruto fresco o en } \\
\text { mermelada }\end{array}$ & Autoconsumo & PPI \\
\hline Mora Rosada & Recolección & Alimenticio: fresco o en mermelada & Autoconsumo & PPI \\
\hline Nabiza & Recolección & Alimenticio: raíz cocinada & Autoconsumo & España - PPI \\
\hline Nabo & Cultivo & Alimenticio: raíz rallada o hervida & Autoconsumo & $\begin{array}{l}\text { España - } \\
\text { Mendoza - PPI }\end{array}$ \\
\hline Níspero & Recolección & $\begin{array}{l}\text { Alimenticio: fruta fresca o en } \\
\text { conservas dulces }\end{array}$ & Autoconsumo & España \\
\hline $\begin{array}{l}\text { Nuez de } \\
\text { Castilla }\end{array}$ & Recolección & Alimenticio & Autoconsumo & España \\
\hline Olivo & $\begin{array}{l}\text { Recolección } \\
\text { (de tierras } \\
\text { públicas/ } \\
\text { comunitarias } \\
\text { en España } \\
\text { y de tierras } \\
\text { privadas en } \\
\text { Mendoza) }\end{array}$ & $\begin{array}{l}\text { Medicinal: para facilitar el sueño, } \\
\text { tranquilizante (hojas) y alimenticio } \\
\text { (fruto) }\end{array}$ & Autoconsumo & $\begin{array}{l}\text { España - } \\
\text { Mendoza }\end{array}$ \\
\hline Orégano & $\begin{array}{l}\text { Recolección } \\
\text { Cultivo }\end{array}$ & $\begin{array}{l}\text { Condimento } \\
\text { Medicinal }\end{array}$ & Comercialización & España \\
\hline Ortiga & Recolección & Alimenticio: hojas hervidas & Autoconsumo & España \\
\hline Papa & Cultivo & Alimenticio & Autoconsumo & $\begin{array}{l}\text { España - } \\
\text { Mendoza }\end{array}$ \\
\hline Pepino & Cultivo & Alimenticio & Autoconsumo & $\begin{array}{l}\text { España - } \\
\text { Mendoza }\end{array}$ \\
\hline Pera & Recolección & $\begin{array}{l}\text { Alimenticio: fruta fresca, seca o en } \\
\text { conservas dulces }\end{array}$ & $\begin{array}{l}\text { Autoconsumo } \\
\text { Trabajo } \\
\text { asalariado: } \\
\text { en cosecha } \\
\text { y en fábrica } \\
\text { de conservas } \\
\text { (Mendoza) }\end{array}$ & $\begin{array}{l}\text { España - } \\
\text { Mendoza }\end{array}$ \\
\hline Perejil & Cultivo & Alimenticio/ condimento & Autoconsumo & $\begin{array}{l}\text { España - } \\
\text { Mendoza - PPI }\end{array}$ \\
\hline $\begin{array}{l}\text { Pimiento } \\
\text { Amarillo o } \\
\text { Banana }\end{array}$ & Cultivo & Alimenticio: fresco & Autoconsumo & Mendoza \\
\hline $\begin{array}{l}\text { Pimiento } \\
\text { Cacho de } \\
\text { cabra }\end{array}$ & Cultivo & Alimenticio: seco & Autoconsumo & Mendoza - PPI \\
\hline
\end{tabular}




\begin{tabular}{|c|c|c|c|c|}
\hline $\begin{array}{l}\text { Nombre } \\
\text { atribuido }\end{array}$ & $\begin{array}{l}\text { Modo de } \\
\text { obtención }\end{array}$ & Formas de utilización & Destino & Lugar de uso \\
\hline $\begin{array}{l}\text { Pimiento } \\
\text { Calahorra }\end{array}$ & Cultivo & Alimenticio: fresco o en conservas & $\begin{array}{l}\text { Autoconsumo } \\
\text {-Comercialización }\end{array}$ & Mendoza - PPI \\
\hline $\begin{array}{l}\text { Pimiento } \\
\text { Carnoso }\end{array}$ & Cultivo & $\begin{array}{l}\text { Alimenticio: para secar y hacer } \\
\text { pimentón dulce }\end{array}$ & Autoconsumo & Mendoza \\
\hline $\begin{array}{l}\text { Pimiento } \\
\text { Largo }\end{array}$ & Cultivo & Alimenticio & Autoconsumo & Mendoza \\
\hline $\begin{array}{l}\text { Pimiento } \\
\text { Medio Kilo }\end{array}$ & Cultivo & $\begin{array}{l}\text { Alimenticio: para secar y hacer } \\
\text { pimentón dulce }\end{array}$ & Autoconsumo & Mendoza \\
\hline $\begin{array}{l}\text { Pimiento o } \\
\text { Morrón dulce }\end{array}$ & Cultivo & Alimenticio & Autoconsumo & España \\
\hline Poroto alubia & $\begin{array}{l}\text { Entrega por } \\
\text { parte del } \\
\text { gobierno }\end{array}$ & Alimenticio: en guisos & Autoconsumo & España \\
\hline Poroto blanco & $\begin{array}{l}\text { Entrega por } \\
\text { parte del } \\
\text { gobierno }\end{array}$ & Alimenticio: en guisos & Autoconsumo & España \\
\hline Poroto negro & $\begin{array}{l}\text { Entrega por } \\
\text { parte del } \\
\text { gobierno }\end{array}$ & Alimenticio: en guisos & Autoconsumo & España \\
\hline Remolacha & Cultivo & Alimenticio & Autoconsumo & $\begin{array}{l}\text { España - } \\
\text { Mendoza }\end{array}$ \\
\hline Roble & Recolección & $\begin{array}{l}\text { Medicinal: para problemas } \\
\text { respiratorios, expectorante en jarabe }\end{array}$ & Autoconsumo & Mendoza - PPI \\
\hline Romero & $\begin{array}{l}\text { Recolección } \\
\text { Cultivo }\end{array}$ & $\begin{array}{l}\text { Condimento. Medicinal. Ahuyentar } \\
\text { insectos en huerta }\end{array}$ & $\begin{array}{l}\text { Autoconsumo } \\
\text { Comercialización }\end{array}$ & $\begin{array}{l}\text { España - } \\
\text { Mendoza }\end{array}$ \\
\hline Sauco & Recolección & Alimenticio: fresco o en mermelada & Autoconsumo & PPI \\
\hline Sertal & Cultivo & Medicinal: para problemas digestivos & Autoconsumo & PPI \\
\hline Tomate & Cultivo & Alimenticio & Autoconsumo & $\begin{array}{l}\text { España - } \\
\text { Mendoza - PPI }\end{array}$ \\
\hline $\begin{array}{l}\text { Tomate } \\
\text { Amarillo }\end{array}$ & Cultivo & Alimenticio: fresco o en conservas & Autoconsumo & Mendoza - PPI \\
\hline $\begin{array}{l}\text { Tomate de } \\
\text { Racimo }\end{array}$ & Cultivo & Alimenticio: fresco (en invierno) & Autoconsumo & Mendoza \\
\hline Tomate Perita & Cultivo & $\begin{array}{l}\text { Alimenticio: fresco, seco o en } \\
\text { conservas }\end{array}$ & $\begin{array}{l}\text { Autoconsumo } \\
\text { Comercialización }\end{array}$ & Mendoza - PPI \\
\hline $\begin{array}{l}\text { Tomate Río } \\
\text { Grande }\end{array}$ & Cultivo & Alimenticio: en conservas & $\begin{array}{l}\text { Autoconsumo } \\
\text { Comercialización }\end{array}$ & Mendoza - PPI \\
\hline Tomillo & $\begin{array}{l}\text { Recolección } \\
\text { Cultivo }\end{array}$ & $\begin{array}{l}\text { Medicinal: té para problemas de } \\
\text { riñones. Condimento }\end{array}$ & $\begin{array}{l}\text { Autoconsumo } \\
\text { Comercialización }\end{array}$ & $\begin{array}{l}\text { España - } \\
\text { Mendoza }\end{array}$ \\
\hline Trigo & $\begin{array}{l}\text { Entrega por } \\
\text { parte del } \\
\text { gobierno }\end{array}$ & Alimenticio: en guisos & Autoconsumo & España \\
\hline Uva & $\begin{array}{l}\text { Recolección } \\
\text { Cultivo }\end{array}$ & $\begin{array}{l}\text { Alimenticio: fruta fresca, seca, en } \\
\text { conservas dulces o preparación de } \\
\text { vino }\end{array}$ & $\begin{array}{l}\text { Autoconsumo } \\
\text { Comercialización } \\
\text { Trabajo } \\
\text { asalariado: } \\
\text { en cosecha } \\
\text { (Mendoza) }\end{array}$ & $\begin{array}{l}\text { España - } \\
\text { Mendoza }\end{array}$ \\
\hline
\end{tabular}




\begin{tabular}{|c|c|c|c|c|}
\hline $\begin{array}{l}\text { Nombre } \\
\text { atribuido }\end{array}$ & $\begin{array}{l}\text { Modo de } \\
\text { obtención }\end{array}$ & Formas de utilización & Destino & Lugar de uso \\
\hline Verdolaga & Recolección & $\begin{array}{l}\text { Alimenticio: en ensalada, guiso y } \\
\text { tortilla }\end{array}$ & Autoconsumo & Mendoza - PPI \\
\hline Zapallo batata & Cultivo & Alimenticio & Autoconsumo & Mendoza - PPI \\
\hline $\begin{array}{l}\text { Zapallo } \\
\text { Cantimplora }\end{array}$ & Cultivo & $\begin{array}{l}\text { Alimenticio } \\
\text { Como utensilio: para cargar agua }\end{array}$ & Autoconsumo & $\begin{array}{l}\text { España - } \\
\text { Mendoza - PPI }\end{array}$ \\
\hline $\begin{array}{l}\text { Zapallo color } \\
\text { verde oscuro }\end{array}$ & Cultivo & Alimenticio & Autoconsumo & $\begin{array}{l}\text { España - } \\
\text { Mendoza - PPI }\end{array}$ \\
\hline $\begin{array}{l}\text { Zapallo de } \\
\text { brujita }\end{array}$ & Cultivo & $\begin{array}{l}\text { Alimenticio } \\
\text { Como utensilio: plato }\end{array}$ & Autoconsumo & $\begin{array}{l}\text { España - } \\
\text { Mendoza - PPI }\end{array}$ \\
\hline Zapallo plomo & Cultivo & Alimenticio & Autoconsumo & Mendoza - PPI \\
\hline Zarzamora & Recolección & $\begin{array}{l}\text { Alimenticio: fruta fresca o en } \\
\text { conservas dulces }\end{array}$ & Autoconsumo & España \\
\hline
\end{tabular}

\section{Discusión y Conclusiones}

La comparación de las estrategias metodológicas aplicadas permitió ratificar la existencia de una "cosecha oculta", orientada por conocimientos botánicos que pueden ponerse en juego en distintos momentos y contextos, ampliando la diversidad biocultural local. De un total de 159 taxones registrados, se han observado y coleccionado 131 especies o variedades, y 28 taxones evocados. Es destacable que el número de taxones referidos en la trayectoria de vida fue muy próximo (99 especies o variedades de 30 familias botánicas) al obtenido mediante observación y realización de entrevistas (98 especies o variedades de 28 familias botánicas). Esto contrasta con trabajos realizados en huertos comerciales, que tienen un manejo familiar en la misma área de estudio. En estos huertos, Bonicatto et al. (2015) relevaron 179 variedades comerciales diferentes correspondientes a 14 familias botánicas (es decir, mayor diversidad comercial de menor número de variedades).

Desde el punto de vista cualitativo se observa también cómo el estudio de la trayectoria de vida ha permitido el registro de una elevada biodiversidad de cultivos de familias botánicas de gran importancia económica, como Cucurbitáceas, Solanáceas y Leguminosas, aumentando la biodiversidad registrada mediante las estrategias sincrónicas.
Sin embargo, es menor el número de Brasicáceas que en otros trabajos (Bonicatto et al., 2015; Pochettino et al., 2014), posiblemente como consecuencia del origen español de la interlocutora, por contraposición al origen mayormente italiano del resto de los entrevistados. Sin embargo, al igual que en los trabajos mencionados y otros sobre el rol de la horticultura (Calvet Mir et al., 2014), se destaca que los criterios de afectividad ligados a la tradición familiar son importantes en dicha conservación. En el caso estudiado tampoco se señala ninguna de las variedades de cultivo típicas del Parque Pereyra Iraola, registradas en este trabajo y en trabajos previos (Del Río et al., 2007; Pochettino, 2010), como el "zapallito lustroso", el "hinojo de cabeza gigante" y la "acelga de penca verde". Se estima que la no incorporación de variedades típicas se debe a su relativamente corta persistencia en el área, ya que la participante está asentada de forma permanente en el Parque Pereyra Iraola desde el año 2015. En este sentido, si bien el caso no es completamente representativo de la población local, dada su corta permanencia en el PPI, en concordancia con una de las dificultades planteadas en la metodología, aporta resultados novedosos que contribuyen como una voz más a la diversidad biocultural local, al ser contrastados con datos del área obtenidos a través de otras estrategias metodológicas, y dan respuesta a las preguntas de investigación planteadas. 
También cabe resaltar que la técnica de la trayectoria de vida ha permitido a la propia interlocutora rememorar y actualizar sus experiencias. De las 99 etnoespecies reconocidas, 58 fueron aprendidas en España; 30 en Mendoza, y 11 en el periurbano platense (Parque Pereyra Iraola), destacándose entonces España como el espacio de primera socialización y de adquisición de mayor diversidad de saberes. Con respecto a las continuidades y discontinuidades entre los lugares de uso, en primer término la relación entre España y Mendoza implicó no sólo el traslado de conocimientos hacia la Argentina, sino también de material genético. Pero los conocimientos inicialmente adquiridos le permitieron reconocer plantas que no eran comúnmente utilizadas en la nueva localización, como el caso del hinojo espontáneo. Mendoza, por su parte, representó el espacio de la diversificación hortícola, dado que por la actividad laboral desarrollada, así como por intercambio con otros productores, la interlocutora tuvo estrecho contacto con distintas variedades de cultivo de tomates, pimientos y zapallos. La aplicación de estos conocimientos en su nueva residencia en la huerta del Parque Pereyra Iraola implicó la incorporación de nuevas variedades al repertorio de la horticultura local. Un caso muy interesante resulta de la comparación de los saberes adquiridos en España y aplicados directamente en la última residencia, dado que las plantas que aparecen en el relato son, en su mayoría, plantas espontáneas obtenidas por recolección. Este es el caso de los cardos, resignificados en la actualidad por su valor emotivo ligado a la historia familiar, pero no tan utilizados como en España, reproduciendo un proceso general ya descripto por Hernández Bermejo et al. (2019).

En este marco, los saberes pueden permanecer latentes: los conocimientos que por múltiples circunstancias no pueden ponerse en práctica en determinados contextos, quedan a la espera de poder expresarse y emerger en contextos propicios. Esta observación ya fue realizada por Nazarea (1998), quien describe los saberes hortícolas de mujeres migrantes entre el campo y la ciudad, destacando que quienes tuvieron posibilidades de acceder al mercado, revierten esa experiencia sobre la diversidad hortícola local.

De acuerdo con los resultados, consideramos que la aplicación de estudios biográficos tiene una serie de ventajas: 1) conocer con mayor detalle el punto de vista del actor local; 2) hacer evidente la dimensión temporal en el escenario de la diversidad biocultural local; 3) dar cuenta de cómo se actualizan/resignifican saberes adquiridos en un contexto particular en otro nuevo; 4) posibilitar un espacio de reflexión para la persona que, al relatarle a otro sus vivencias, ordena, resignifica y revaloriza sus propias experiencias y saberes; 5) explicitar el rol activo del investigador: el "cómo" de la investigación se vuelve también parte del "para qué", que incluye la revalorización de los saberes locales. En este punto, se puede agregar que el relato obtenido, en formato escrito, fue entregado a la interlocutora, quien, a su vez, solicitó varias copias para repartir entre personas allegadas.

Asimismo, no se desconocen los problemas que plantean los estudios biográficos, a saber: 1) selección adecuada del interlocutor a los fines de la investigación; en nuestro caso, sobre la base del conocimiento previo del área y los pobladores locales se seleccionó una interlocutora con voluntad y capacidad de participación, así como experiencia en la actividad hortícola, a pesar de su corta permanencia en la zona; 2) se requiere más tiempo para su implementación y registro; 3) cuán representativo es el interlocutor del grupo social o de la población de estudio; en este sentido no se niega la poca representatividad de emplear un solo caso, pero en tanto complemento de los datos aportados por otras estrategias metodológicas se considera que aporta al conocimiento de la diversidad biocultural local. En el caso presentado se destaca que mediante una única técnica (historia de vida) y con una sola persona se obtuvieron referencias a 99 etnoespecies, mientras que utilizando técnicas sincrónicas de observación y entrevista, con 25 personas, se identificaron 98 etnoespecies; 4) no obstante, también se destaca la imposibilidad para poder contrastar lo que se nos dice con la observación de material vegetal en lo que se refiere al relato del pasado. El uso de diversos 
estímulos visuales y narrativos constituye una herramienta útil, pero no conclusiva.

En este marco, entendemos que la integración de metodologías sincrónicas de entrevista y observación con los estudios biográficos resulta en un abordaje integral y complejo de la diversidad biocultural que no podría haberse alcanzado por una única vía metodológica. Asimismo, aportan valiosa información de aplicación inmediata para proyectos aplicados y de desarrollo local, de los cuales los huertos familiares suelen ser protagonistas centrales.

\section{Agradecimientos}

Los autores agradecen a los participantes locales por su generosidad en compartir tiempo y saberes, y permitir su difusión; y a los revisores anónimos, por sus aportes y sugerencias. Esta investigación fue realizada con apoyo económico de la UNLP, CONICET y FONCyT (PICT 2015-1548), y el estudio de trayectoria de vida es resultado de la beca CIN otorgada a Ana Paula Castello.

\section{Bibliografía}

Albuquerque, U. P., Andrade, L. \& Caballero, J. (2005). Structure and floristics of homegardens in Northeastern Brazil. Journal of Arid Environments 62: 491-506. https://doi.org/10.1016/j.jaridenv.2005.01.003.

Albuquerque, U. P., Cruz da Cunha, L., Lucena, R. \& Alves, R. (eds). (2014). Methods and techniques in ethnobiology and ethnoecology. Springer Protocols Handbooks. Humana Press, New York.

Albuquerque, U. P., Lucena, R., Cruz da Cunha, L. \& Alves, R. (eds). (2019). Methods and techniques in ethnobiology and ethnoecology. 2nd edition. Springer Protocols Handbooks. Humana Press, New York. https://doi.org/10.1007/978-1-4939-8919-5

BARSKY, A. (2010). La agricultura de "cercanías" a la ciudad y los ciclos del territorio periurbano. Reflexiones sobre el caso de la Región Metropolitana de Buenos Aires. En Svetlitza de Nemirovsky, A. (ed.), Agricultura periurbana en Argentina y globalización. Escenarios, recorridos y problemas, pp. 15-29. FLACSO, Buenos Aires.
Benencia, R. (1997). Área hortícola Bonaerense. Cambios en la producción y su incidencia en los sectores sociales. La Colmena, Buenos Aires.

Bertaux, D. (1999). El enfoque biográfico: su validez metodológica, sus potencialidades. Proposiciones 29: 1-22. http://www.sitiosur.cl/publicaciones/ Revista_Proposiciones/PR-0029-3258.pdf.

Blanckaert, I., Swennen, R., Paredes, M., Rosas, R. \& Lira SAade, R. (2004). Floristic composition, plant uses and management practices in homegardens of San Rafael Coxcatlán, Valley of Tehuacán, Mexico. Journal of Arid Environments 57: 179-202. https://doi.org/10.1016/S0140-1963(03)00100-9

Bonicatto, M. M., Marasas, M. E., Sarandon, S. \& Pochettino, M. L. (2015). Seed conservation by family farmers in the rural-urban fringe area of La Plata Region, Argentina: the dynamics of an ancient practice. Agroecology and Sustainable Food Systems 39: 625-646. https://doi.org/10.1080/21683565.2015.1020405

Buet Costantino, F., Ulibarri, E. A. \& Hurrell, J. A. (2010). Las huertas familiares en la isla Paulino (Buenos Aires, Argentina). En Pochettino, M. L., Ladio, A. H. \& P. M. Arenas (eds.), Tradiciones y Transformaciones en Etnobotánica, pp. 479-484. CYTED-RISAPRET, San Salvador de Jujuy.

Calvet-Mir, L., Calvet-Mir, M., Vaqué-Nuñez, L. \& Reyes-García, V. (2011). Landraces in situ conservation: a case study in high-mountain home gardens in VallFosca, Catalan Pyrenees, Iberian Peninsula. Economic Botany 65: 146-157. https://doi.org/10.1007/s12231-011-9156-1

Calvet-Mir, L., Garnatje, T., Parada, M., Vallès, J. \& REYES-GARCÍA, V. (2014). Más allá de la producción de alimentos: los huertos familiares como reservorios de diversidad biocultural. Ambienta (España) 107: 40-53.

Campbell, B. M. \& Luckert, M. K. (2002). Evaluando la cosecha oculta de los bosques. Nordan Comunidad, Montevideo.

DAs, T. \& DAs, A. K. (2005). Inventorying plant biodiversity in homegardens. A case study in Barak Valley, Assam, North East India. Current Science 89: 155-163. https://www.jstor.org/ stable/24110441.

Del Río, J. P., Maidana, J. A, Molteni, A., Pérez, M., Pochettino, M. L., Souilla, L., Tito, G. \& Turco, E. (2007). El rol de las "quintas" familiares del Parque Pereyra Iraola (Buenos Aires, Argentina) en la conservación de la agrobiodiversidad. Kurtziana 33: 217-226. 
Ferto, M. C. (2007). Modalidades de intervención social sobre los horticultores bonaerenses: una mirada antropológica. Avá (Posadas) 10: 78-96.

Ferraroti, F. (2007). Las historias de vida como método. Convergencia. Revista de Ciencias Sociales 14: 15-40. https://www.redalyc.org/articulo. oa?id=105/10504402.

GARCíA, M. (2010). Inicios, consolidación y diferenciación de la horticultura platense. En Svetlitza DE Nemirovsky, A. (ed.), Agricultura periurbana en Argentina y globalización. Escenarios, recorridos y problemas, pp. 69-85. FLACSO, Buenos Aires.

Gaytán, C., Vibrans, H., Navarro, H. \& Jiménez, M. (2001). Manejo de huertos familiares periurbanos de San Miguel Tlaixpan, Texcoco, México. Boletín de la Sociedad Botánica de México 69: 32-62. https://doi.org/10.17129/botsci.1646

Gómez Sal, A., González García A. \& Doña, H. (2014). La cultura del patio como soporte de agricultura familiar en América Tropical. Ambienta (España) 107: 74-85.

Hernández-Bermejo, J. E., Delucchi, G., Charra, G., Pochettino, M. L. \& Hurrell, J. A. (2019). "Cardos" of two worlds: Transfer and re-signification of the uses of thistles between the Iberian Peninsula and Argentina. Ethnobiology and Conservation 8: 5. https://doi.org/10.15451/ec2019-03-8.05-1-22

Huai, H. \& Hamilton, A. (2009). Characteristics and functions of traditional homegardens: A review. Frontiers of Biology in China 4: 151-157. https://doi.org/10.1007/s11515-008-0103-1

Hurrell, J. A., Buet Costantino, F., Puentes, J. P., Ulibarri, E. A. \& Pochettino, M. L. (2011). Huertos familiares periurbanos de las costas de EnsenadaBerisso y de la Isla Martín García (Buenos Aires, Argentina). Bonplandia 20: 213-229.

https://doi.org/10.30972/bon.2021325

Hurrell, J. A., Cabanillas, P. A., Guerrero, E. L. \& Delucchi, G. (2014). Naturalización y etnobotánica de Vitis labrusca L. (Vitaceae) en la región rioplatense, Argentina. Revista Museo Argentino Ciencias Naturales (nueva serie) 16: 13-18. https://doi.org/10.22179/REVMACN.16.158

Lamont, S., Hardy Eshbaugh, W. \& Greenberg, A. (1999). Species composition, diversity, and use of homegardens among three Amazonian villages. Economic Botany 53: 312-326. https://doi.org/10.1007/BF02866644

Lema, V. S. (2006). Huertos de altura: el manejo humano de especies vegetales en la puna argentina. Revista de la Escuela de Antropología (Rosario) 12: 173-186.
Llobera Serra, P. (2014). Horticultura urbana: La red de huertos urbanos comunitarios de Madrid. Ambienta (España) 107: 120-128.

Marasas, M. \& Velarde, I. (2000). Rescate del saber tradicional como estrategia de desarrollo: los viñateros de la costa. Boletín ILEIA 16: 23-24.

Martínez, M. R., Pochettino, M. L. \& Arenas, P. M. (2003). La horticultura: estrategia de subsistencia en contextos pluriculturales, Valle del Cuñapirú, Misiones, Argentina. Delpinoa 45: 89-98.

Montañez-Escalante, P. I., Ruenes-Morales, M. R., Ferrer-Ortega, M. M. \& Estrada-Medina, H. (2014). Los huertos familiares Maya-Yucatecos: situación actual y perspectivas en México. Ambienta (España) 107: 6-18.

Moyano Estrada, E. (2014). La agricultura familiar revisitada. Una mirada a la agricultura como factor de desarrollo social y económico. Ambienta (España) 107: 100-109.

NAZAREA, V. D. (1998). Cultural memory and biodiversity. University of Arizona Press, Tucson.

Pamungkas, R. N., Indriyani, S. \& Hakim, L. (2013). The ethnobotany of homegardens along rural corridors as a basis for ecotourism planning: a case study of Rajegwesi village, Banyuwangi, Indonesia. Journal of Biodiversity and Environmental Sciences 3: 60-69.

Pochettino, M. L. (2010). Huertos periurbanos como aporte a la diversidad agrícola, Provincia de Buenos Aires, Argentina. En Pochettino, M. L., A. H. Ladio \& P. M. Arenas (eds.), Tradiciones y Transformaciones en Etnobotánica (ICEB 2009), pp. 186-192. CYTEDRISAPRET, San Salvador de Jujuy.

Pochettino, M. L., Souilla, L. \& Turco, E. (2006). Adaptación a nuevas condiciones sociales $\mathrm{y}$ económicas entre los viejos "quinteros" del Parque Pereyra Iraola (Buenos Aires). VIII Congreso de Antropología Social, Simposio (CD), Salta, Argentina.

Pochettino, M. L., Hurrell, J. A. \& Lema, V. S. (2012). Local botanical knowledge and Agrobiodiversity: Homegardens at rural and periurban contexts in Argentina. En Luna Maldonado, A. I (ed.), Horticulture, pp. 105-132. InTech, Rijeka, Croacia.

Pochettino, M. L., Hurrell, J. A. \& Bonicatto, M. M. (2014). Horticultura periurbana: estudios etnobotánicos en huertos familiares de la Argentina. Ambienta (España) 107: 86-99.

Pochettino, M. L., Bonicatto M. M. \& Hurrell, J. A. (2017). Huertos comerciales del periurbano de La Plata (Buenos Aires, Argentina): Reservorio de diversidad biocultural. En A. CASAS, J. Torres 
Guevara \& F. Parra (eds.), Domesticación en el continente americano 2, pp. 413-430. Universidad Nacional Autónoma de México, Morelia.

Reyes-Garcia, V., Aceituno, L., Vila, S., Calvet-Mir, L., Garnatje, T., Jesch, A., Lastra, J. J., Parada, M., Rigat, M., Vallès, J. \& Pardo de Santayana, M. (2012). Home gardens in three mountain regions of the Iberian Peninsula: Description, motivation for gardening, and gross financial benefits. Journal of Sustainable Agriculture 36: 249-270. https://doi.org/10.1080/10440046.2011.627987

Seta, T., Demissew, S. \& Asfa, Z. (2013). Home gardens of Wolayta, Southern Ethiopia. An ethnobotanical profile. Academia Journal of Medicinal Plants 1: 14-30. http://dx.doi.org/10.15413/ajmp.2012.0108

The Plant List. (2013). The Plant List. Version 1.1. Disponible: http://www.theplantlist.org/ (Consulta: 5/2020).

Turco, E., Souilla, L. \& Pochettino, M. L. (2006). Relación entre saberes y prácticas culinarios con la conservación de la agrobiodiversidad. Estudio etnobotánico en el Parque Pereyra Iraola (Buenos Aires, Argentina). VII Congreso Latinoamericano de Sociología Rural (CD). Quito, Ecuador.

Velarde, I., Voget, C., Avila, G., Loviso, C., Orosco, E., Sepúlveda, C. \& Artaza, S. (2008). Influencia de la calidad en el consumo de productos patrimoniales: el caso del sistema agroalimentario del vino de la costa de Berisso. En Velarde, I., A. Maggio \& J. OTERo (eds.), Sistemas agroalimentarios localizados en Argentina, pp. 31- 66. Instituto Nacional de Tecnología Agropecuaria, Buenos Aires.
Vlkova, M., Polesny, Z., Verner, V., Banout, J., Dvorak, M., Havlik, J., Lojka, B., Ehl, P. \& Krausova, J. (2010). Ethnobotanical knowledge and agrobiodiversity in subsistence farming: case study of home gardens in Phong My commune, central Vietnam. Genetic Resources and Crop Evolution, https://doi.org/10.1007/s10722-010-9603-3

Vogl, C. R., Vogl-Lukasser, B. \& Caballero, J. (2002). Homegardens of Maya migrants in the Palenque District (Chiapas/Mexico). Implications for sustainable rural development. En Stepp, J. R., F. S. Wyndham \& R. K. ZARger (eds.), Ethnobiology and Biocultural Diversity, pp. 631- 647. University of Georgia Press, Athens.

Vogl-LuKasser, B. \& Vogl, C. R. (2004). Ethnobotanical research in homegardens of small farmers in the Alpine region of Osttirol (Austria). An example for bridges built and building bridges. Ethnobotany Research \& Applications 2: 111-137. https://doi.org/10.17348/era.2.0.111-137

Vogl-LuKasser, B., Vogl C. R. \& Bolhàr-NordenKampF, H. (2002). Homegarden composition on small peasant farms in the Alpine regions of Eastern Tyrol (Austria) and their role in sustainable rural development. En Stepp, J. R., F. S. Wyndham \& R. K. ZARGER (eds.), Ethnobiology and Biocultural Diversity, pp. 648-658. University of Georgia Press, Athens.

Watson, J. W. \& Eyzaguirre, P. B. (eds.). (2002). Home gardens and in situ conservation of plant genetic resources in farming systems. International Plant Genetic Resources Institute, Rome. 